\title{
LA COMPOSICIÓN DINÁSTICA DE YAXCHILÁN DURANTE EL REINADO DE YAXUUN B'AHLAM IV
}

\author{
María Elena Vega Villalobos \\ Maestría en Estudios Mesoamericanos \\ Facultad de Filosofía y Letras, UNAM
}

Yitaaj Cha' Mo' Ajaw

\section{Introducción}

La historia de la antigua ciudad de Yaxchilán ${ }^{1}$ no termina con el abandono del sitio en el siglo x d.C. Los habitantes de la zona, los lacandones, aún consideran sagrado el lugar y sus monumentos; los distintos visitantes del siglo xIx (mexicanos, europeos, estadounidenses) exploraron y revelaron al mundo la calidad de sus esculturas; los arqueólogos y demás estudiosos durante el siglo xx establecieron secuencias ocupacionales, fecharon los distintos monumentos y, a partir de la segunda mitad del siglo $\mathrm{xx}$, los nombres de los personajes representados en las inscripciones y sus hazañas comenzaron a conocerse (Proskouriakoff, 1963, 1964).

De esta forma, la arqueología, la historia y la epigrafía nos revelan cada día nuevos datos que nos permiten conocer y entender un poco más el mundo maya de Yaxchilán. El hallazgo de nuevos monumentos, las exploraciones arqueológicas y el conocimiento cada vez más profundo de las crónicas y documentos coloniales presentan un reto a los investigadores en cuanto a interpretaciones epigráficas y explicaciones en torno a la organización sociopolítica y al pensamiento religioso.

Si bien es cierto que las inscripciones jeroglíficas sólo nos hablan de una pequeña parte de la sociedad, la elite, no por ello son menos importantes. La epigrafía tiene, entre muchas otras ventajas, la de hacer evidente el discurso histórico que los antiguos mayas dejaron escrito.

\section{Datos generales}

La zona arqueológica de Yaxchilán (figura 1) se encuentra ubicada sobre el margen izquierdo del río Usumacinta, en la selva Lacandona, en el estado de Chia-

\footnotetext{
${ }^{1}$ Simon Martin (2004) ha propuesto que el glifo emblema de Yaxchilán debió leerse como k'uhul $P a$ ' Chan ajaw, "señor sagrado del Cielo Partido".
} 


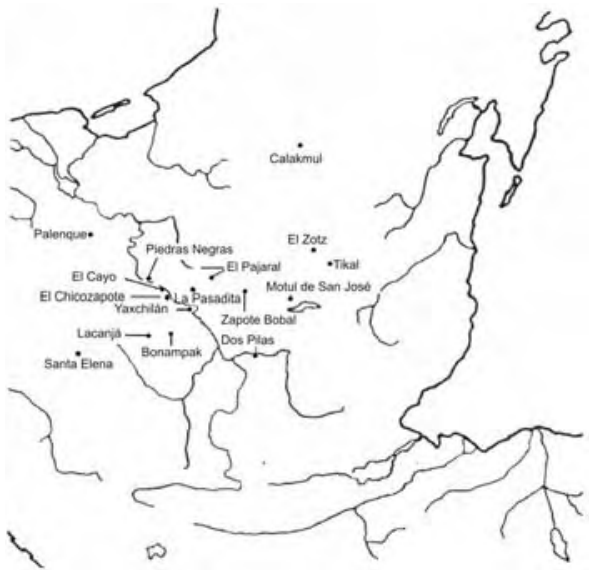

Figura 1. Mapa que muestra la ubicación de Yaxchilán y los sitios con los que tuvo contacto

pas. El sitio cuenta, en su área central, con 120 construcciones distribuidas en tres grandes conjuntos: la Gran Plaza, ubicada en la parte baja de la ciudad y paralela al río; la Gran Acrópolis y la Pequeña Acrópolis, adaptadas mediante terrazas y plataformas (figura 2). Los tres conjuntos están conectados a través de escalinatas, rampas y terrazas de distribución (Sotelo Santos, 1992; Brokmann, 2000; García Moll, 2003). Hasta ahora, los monumentos esculpidos de Yaxchilán suman más de 110. La mayoría corresponde al Clásico Tardío, es decir, entre el 681 y el 808 d.C. (Mathews, 1997; Martin y Grube, 2002).

Las exploraciones arqueológicas (García Moll, 2004) permiten establecer que el sitio estuvo habitado durante más de mil años (300 a.C.-900 d. C.). Las primeras construcciones se han fechado entre los siglos in y vi d.C. Estas construcciones (edificios 7-Sub, 18, 77 y 78) son grandes basamentos que delimitan el lado oeste de lo que llegará a ser la Gran Plaza. 


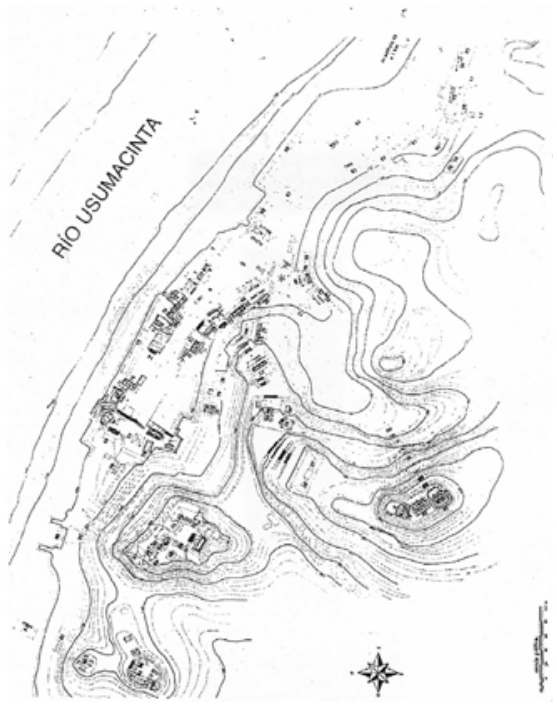

Figura 2. Mapa de la ciudad de Yaxchilán. Tomado de García Moll, 2003

Antes de entrar a los textos de Yaxuun B’ahlam IV y a la composición dinástica del señorío durante su reinado, es necesario hablar de la historia de Yaxchilán durante el Clásico Temprano. La mención de los distintos gobernantes será muy breve, de tal suerte que la información se organizará y simplificará para resumir en unas cuantas páginas un siglo. 


\section{Los gobernantes de Yaxchilán antes de Yaxuun B'ahlam IV: la historia dinástica}

Los textos de Yaxchilán nos dicen que el fundador del linaje, Yopaat B'ahlam, se entronizó en el año 359 d.C. Los monumentos donde se escribió su entronización son la Escalera Jeroglífica 1, con una fecha en Rueda Calendárica de 7 Imix 14 Sootz' (Mathews, 1997), y el Dintel 11, sin fecha.

La historia dinástica de Yaxchilán se ha reconstruido a partir de cuatro dinteles del periodo Clásico Temprano: el 11, el 49, el 37 y el 35 . En ellos encontramos diez ascensiones al trono. Las fechas en Rueda de Calendario y Cuenta Larga han sido establecidas por Peter Mathews en su tesis doctoral (1997). De esta forma, podemos ubicar a los primeros diez gobernantes del sitio entre 8.16.2.9.1 (359 d. C.) y 9.4.11.8.16 (526 d.C.). Roberto García Moll (2003) nos dice que para 300 d.C. ya encontramos en Yaxchilán un asentamiento humano que pudo estar distribuido por toda la zona.

El último registro del periodo Clásico Temprano lo tenemos en el Dintel 35 en Rueda de Calendario: (9.5.2.10.6), 1 Kimi 14 Muan, 537 d.C. Aunque hay otros registros tempranos en el sitio, como los dinteles 19, 20, 22 y 34, debido a la destrucción que han sufrido o a la falta de fechas registradas en ellos es difícil ubicarlos con precisión. Sin embargo, por el estilo y la información sabemos que pertenecen al Clásico Temprano.

A través de los trabajos epigráficos se ha reconocido a dos gobernantes sobresalientes; el primero es Joy B'ahlam, quien accedió al trono en (9.3.13.12.9), 4 Muluk 17 Xul, 28 de julio de 508 d.C., mencionado en dos monumentos tempranos: el Dintel 37 y quizá la Estela 14. El segundo es K'inich Tataab' Joloom; la fecha de su entronización fue escrita en Cuenta Larga con variantes de cabeza y de cuerpo completo en uno de los dinteles más hermosos de la ciudad, el 48 . En este monumento se registra la fecha (9.4.11.8.16), 2 Kib' 19 Pax, 11 de febrero de 526 d.C., como el momento en que K'inich Tataab' Joloom “se sienta o inicia en el señorío".

Joy B'ahlam es mencionado en la vecina ciudad de Piedras Negras, la cual se ubica en el margen derecho del río Usumacinta, a cincuenta kilómetros de Yaxchilán. El monumento donde encontramos registrado a Joy B'ahlam es el Tablero 12, única inscripción que se asocia con el Gobernante C de Piedras Negras (Martin y Grube, 2002; Bíró, 2004). La última fecha del monumento la tenemos en Rueda de Calendario, (9.4.3.10.1), 7 Imix 19 Pop, 19 de abril de 518 d.C., por lo que se puede asumir que esta Rueda de Calendario nos remite a la fecha en que fueron presentados los cuatro personajes arrodillados y con las manos atadas frente al gobernante de Piedras Negras. Los señores son de distintas entidades políticas y uno de ellos es Joy B'ahlam de Pa' Chan.

A K'inich Tataab' Joloom podemos atribuirle varios de los monumentos tempranos, como los dinteles 47, 48, 35 y 37. Después de la fecha 537 d. C., registrada en el Dintel 35, no encontramos otro texto hasta finales del siglo vil, cuando 
aparece Itzamnaaj B'ahlam II. Aunque algunos investigadores prefieran hablar de un hiato en la arqueología más que de un hiato en las inscripciones jeroglíficas, hasta ahora no se conocen textos de Yaxchilán que se ubiquen con certeza entre los siglos vi y vil, es decir, después de K'inich Tataab' Joloom y antes de Itzamnaaj B'ahlam II. Es cierto que hay monumentos que nos hablan de Yaxuun B'ahlam III entre los años 629 y 669 d.C., pero, desde los trabajos de Tatiana Proskouriakoff (1950; 1963; 1964), estos monumentos se han asignado estilísticamente a la época de Yaxuun B'ahlam IV, en el siglo viII.

Este "hiato" en las inscripciones de Yaxchilán resulta muy interesante porque, a diferencia de otras ciudades, como Tikal, durante este periodo de silencio la ciudad crece al doble de su tamaño. ${ }^{2}$ García Moll, en varios de sus trabajos, ubica el siglo vII como uno de los más gloriosos para Yaxchilán en cuanto a crecimiento interno del sitio y al establecimiento de relaciones con otras ciudades. Antes de 600 d.C. encontramos muy pocas construcciones. Después de esa fecha comienzan a erigirse edificios con cresterías, crujías largas y angostas cubiertas por bóvedas con molduras horizontales y decoración modelada en estuco que exhibe mascarones de deidades solares sobre los tableros del segundo cuerpo (García Moll, 2003).

La epigrafía también ha revelado la presencia de un gobernante para este periodo. El personaje es Yaxuun B'ahlam III, padre de Itzamnaaj B'ahlam II. Los monumentos en los que encontramos referencias a Yaxuun B'ahlam III son las estelas 3 y 6 y algunos escalones de la Escalera Jeroglíica 2, todos realizados posteriormente. Es interesante comentar que el primer personaje asociado con el segundo glifo emblema de Yaxchilán, que posiblemente se lee k'uhul Kaaj? ajaw, ${ }^{3}$ es Yaxuun B'ahlam III.

Los registros de este gobernante llegan hasta el año 669 d.C. (9.11.16.10.13.). En 681 encontramos a uno de los gobernantes más conocidos de la ciudad: Itzamnaaj B'ahlam II, del cual aún existen varias interrogantes. Aunque no contamos con un monumento que hable de forma explícita de su ascensión al trono, Mathews (1997) propuso el año 681, (9.12.9.8.1), 5 Imix 4 Mak, como su fecha de entronización, una fecha que encontramos registrada en dos monumentos: el escalón III de la Escalera Jeroglífica 3 y el Dintel 25.

${ }^{2}$ Stephen D. Houston, et al. (2006) han propuesto que este incipiente desarrollo en Yaxchilán se debió a que la dinastía mencionada en los dinteles tempranos fue originaria de El Zotz, una ciudad ubicada en San Miguel la Palotada, Petén, Guatemala (figura 1). Los autores piensan que el hecho de que ambos sitios (Yaxchilán y El Zotz) compartieran el mismo glifo emblema no fue casual. De esta forma, señalan, la dinastía de Yaxchilán surgió de una dinastía foránea que se originó en el Petén. Sin embargo, los trabajos arqueológicos realizados hasta ahora no permiten fechar el asentamiento antes de la fundación de Yaxchilán, es decir, antes de 359 d.C.

${ }^{3}$ El glifo leído en este artículo como kaaj, "pueblo", es dudoso. Sin embargo, se utilizará a lo largo del trabajo con un signo de interrogación. 
El frente de la Estela 12 de Yaxchilán (figura 3) registra la muerte de Itzamnaaj B'ahlam Il en 742 d.C. Según las inscripciones del sitio, el reinado de este gobernante duró 61 años. Durante ese tiempo, Itzamnaaj B'ahlam Il erigió varios dinteles y la Escalera Jeroglífica 3; mantuvo relaciones antagónicas con sitios cercanos como Hix Witz (que tuvo como capitales a El Pajaral y Zapote Bobal), Xukalnaah (Lacanjá) ${ }^{4}$ y Piedras Negras; dominó ciudades como El Chicozapote y La Pasadita (figura 1). Sin embargo, al momento de su muerte encontramos en Yaxchilán un largo periodo de aparente inestabilidad política. Este momento crítico ha sido llamado interregno (742-752 d.C.), y es uno de los periodos más prolongados de ausencia de gobernantes conocidos hasta ahora en la historia de las ciudades del periodo Clásico.

Varios autores (Proskouriakoff, 1963, 1964; Mathews, 1988; Schele y Freidel, 1992; Stuart, 1993; Grube, 1999; Martin y Grube, 2002; Bíró, 2004) han propuesto que durante el interregno hubo una lucha encarnizada entre distintos personajes para acceder al trono de Yaxchilán. Esta interpretación en los últimos años se argumenta con textos de Dos Pilas y Piedras Negras, los cuales registran eventos interesantes de la historia dinástica de Yaxchilán. En Dos Pilas (figura 4) se asienta que en 745 d.C. fue capturado un personaje identificado como K'ab'al Xook, señor de Pa' Chan, el cual debió tener un parentesco cercano con la esposa principal de Itzamnaaj B'ahlam II. ${ }^{5}$ Por otro lado, en la cercana ciudad de Piedras Negras, el Tablero 3 (figura 5) menciona que fue "vista la canoa blanca [de] Yopaat B'ahlam, sagrado señor de Pa' Chan" en 749, personaje que ha sido asociado a dos individuos: al mismo Yaxuun B'ahlam IV y a un gobernante del interregno, del cual no tenemos referencias en las inscripciones de Yaxchilán. En otro pasaje de este monumento (figura 5a) se registró el "estreno en el señorío" (joyajiily ti ajawlel) de un personaje llamado Yaxuun B'ahlam bajo el auspicio de Pawah K'an Ahk II, gobernante de Piedras Negras, en 757 d.C.

Aunque estos eventos han sido considerados en las propuestas interpretativas en torno al interregno, en Yaxchilán no encontramos registros de ellos, pero sí una serie de acontecimientos importantes ocurridos entre 741 y $752 \mathrm{~d}$.C. El primero, en el frente de la Estela 11 en 741, registra a Yaxuun B'ahlam IV y a Itzamnaaj B'ahlam II intercambiando "bastones alados" un año antes de la muerte de este último. El segundo acontecimiento importante (Dintel 59) ocurre en 749 d.C., cuando muere la señora K'ab'al Xook; el tercero se registra dos años después con el nacimiento del hijo de Yaxuun B'ahlam IV, Chelte' Chan K'inich. Proskouriakoff (1963-1964), Mathews (1988), Grube y Martin (2002) y Ayala Falcón (2002) seña-

\footnotetext{
${ }^{4}$ Escribo el nombre del sitio arqueológico de Lacanjá de esta forma para que no se confunda con la toponimia Lakamha' utilizada en Palenque.

${ }^{5}$ La esposa principal de Itzamnaaj B'ahlam II, la señora K'ab'al Xook, fue representada en los dinteles 24,25 y 26 usando títulos importantes que ostentan los gobernantes mayas como el de kaloomte' y b'aahkab', lo cual ha permitido sugerir que la señora Xook gozaba de gran prestigio e importancia política en la dinastía de Yaxchilán.
} 


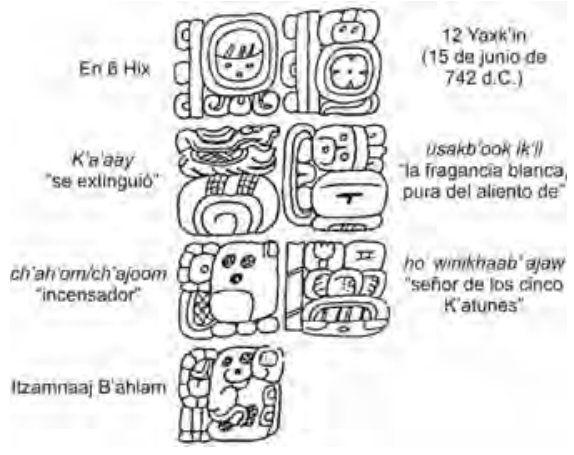

Figura 3. Frente de la Estela 12 de Yaxchilán que registra la muerte de Itzamnaaj B’ahlam. Dibujo de Peter Mathews, 1997

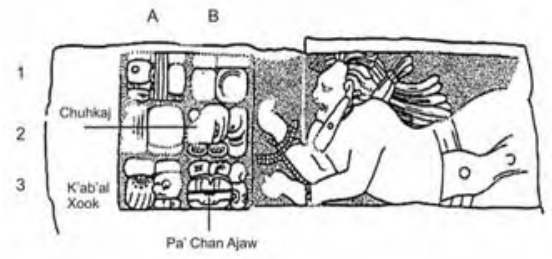

Figura 4. Cautivo identificado como señor de Pa' Chan. Escalera Jeroglífica III, escalón 2 de Dos Pilas. Tomado de Martin y Grube, 2002 
laron que Yaxuun B'ahlam sólo pudo acceder al trono después de la muerte de la señora Xook y del nacimiento de Chelte'. Su inicio en el señorío ocurrió en (9.16.1.0.0, 11), Ajaw 8 Tzek, 29 de abril de 752 d.C., a los 43 años de edad.

\section{Yaxuun B'ahlam IV}

Yaxuun B'ahlam es el primer gobernante de Yaxchilán que se entroniza en una fecha de final de periodo, a diferencia de los primeros diez gobernantes tem-

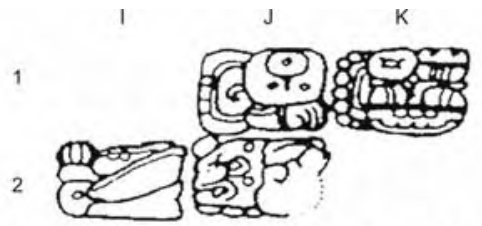

"Fue vista la blanca canoa de Yopaat B'ahlam, señor sagrado de Pa" Chan' Figura 5. Tablero 3 de Piedras Negras. Tomado de Martin y Grube, 2002

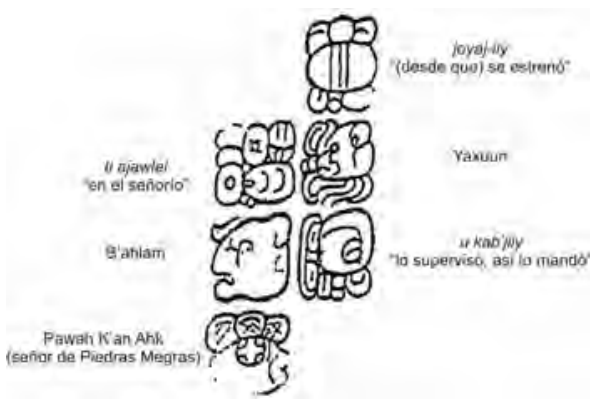

Figura 5a. Texto central del Tablero 3 de Piedras Negras. Tomado de Martin y Grube, 2002 
pranos e Itzamnaaj B'ahlam II. Es durante su reinado que la ciudad alcanza su mayor esplendor: Yaxuun erigió más de la mitad de los monumentos que hoy conocemos: algunas estelas (donde representa a su abuelo Yaxuun B'ahlam III), varios dinteles, las escaleras jeroglíficas 1, 3, 4 y tres altares. A través de estos textos, y sobre todo por los dinteles, sabemos que tuvo cuatro esposas: dos de ellas de Motul de San José, una de Hix Witz y la señora Chak Joloom, una mujer local y madre de Chelte' Chan K'inich.

Yaxuun construyó una gran cantidad de edificios (figura 6). La Estructura 22, en la Gran Plaza, fue el edificio que eligió para honrar la memoria de los gober-

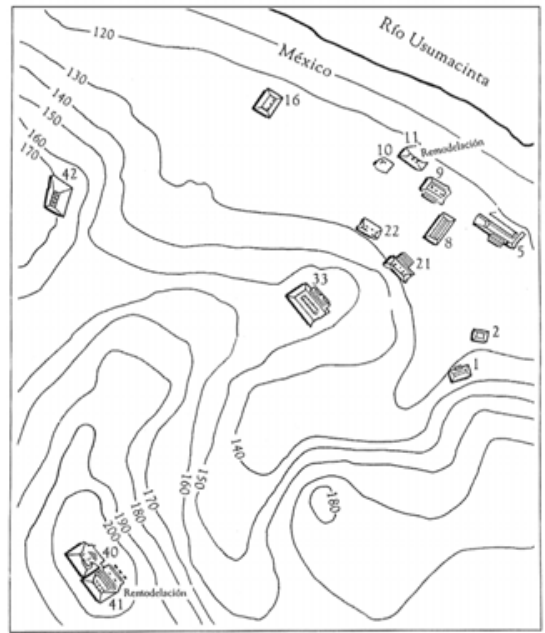

Figura 6. Plano que muestra las estructuras de Yaxuun B'ahlam IV. Tomado de Sotelo Santos,1992 
nantes tempranos del sitio a través de los dinteles $19,20,22$ y, en el vano central de la puerta, el Dintel 21, donde relata un ritual realizado por Cráneo Luna y Yaxuun con más de doscientos años de diferencia. Su gran logro constructivo lo completó con el Edificio 33, una estructura ubicada en el corazón del centro ceremonial. El edificio alberga tres dinteles colocados en sus vanos de acceso y la Escalera Jeroglífica 2.

\section{Un nuevo orden político}

Cuando Yaxuun accedió al trono, la zona del Usumacinta vivía cambios muy significativos. A partir del siglo vil, distintas ciudades comenzaron a representar en sus textos personajes supeditados al k'uhul ajaw, máxima autoridad de las ciudades mayas, los cuales desempeñaban diversas funciones y tenían cargos y jerarquías distintas entre sí (Stuart, 1993; Bernal Romero y Venegas Durán, 2005). Sin embargo, algunos monumentos no sólo mencionan ajawtaak menores en actos de cortejo o comitiva, sino que son presentados como actores únicos de eventos importantes. El ejemplo más claro de ello lo encontramos en el Altar 4 de El Cayo (figura 7), donde el sajal Chak Wayib' K'utiim Ajyax Niil celebra un final de periodo en $(9.15 .0 .0 .0), 4$ Ajaw 13 Yax, 731 d.C., sin la intervención del k'uhul ajaw de Piedras Negras. Acontecimientos parecidos se dan por igual en otras ciudades, sobre todo en Yaxchilán, donde tenemos el registro de diversos actores que conformaron la dinastía durante el reinado de Yaxuun B'ahlam.

Aunque la alusión a ajawtaak menores en Yaxchilán antes de Yaxuun es muy escasa, ${ }^{6}$ sabemos que durante el reinado de Itzamnaaj B'ahlam II existió la figura de sajal por dos referencias: una se encuentra en la Estela 8 de Piedras Negras (figura 8), donde se registró la captura (chuhkaj) del sajal de Itzamnaaj B'ahlam, el supervisor de Ajb'ook. ${ }^{7}$ La otra referencia a un sajal la tenemos en uno de los monumentos del denominado Sitio R (figura 9), donde Itzamnaaj B'ahlam comparte escena con un personaje que usa el título de ajchan b'aak, "el de los cuatro cautivos" llamado Ajkamo', "el de Nuestra Guacamaya”, " y era un ch'ok sajal, "noble sajal" del señor sagrado de Yaxchilán. Esto demuestra que la ausencia de alusiones explícitas a sajales en las inscripciones de Itzamnaaj B'ahlam no implica que no existieran al interior del señorío.

\footnotetext{
${ }^{6}$ Sólo tenemos dos registros de personajes menores: uno está en el Dintel 19, que corresponde al título de ajk'uhu'n, y el otro en el escalón IV de la Escalera Jeroglífica 3, que alude a un sajal.

${ }^{7}$ Eric Velásquez (comunicación personal) me ha señalado que el logograma que se leía NIK ahora se lee B'OK, "fragancia, aliento, perfume".

${ }^{8}$ Eric Velásquez (comunicación personal) propone que el nombre Ajkamo" se traduzca como "el de Nuestra Guacamaya".
} 


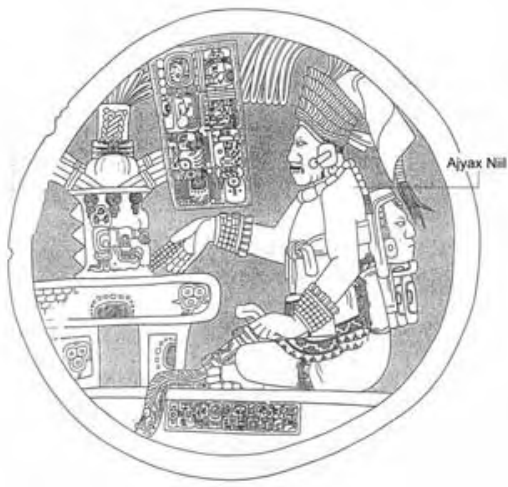

Figura 7. El sajal Ajyax Niil celebrando una fecha de final de periodo. Altar 4 de El Cayo. Dibujo de Peter Mathews, 1997

\section{La composición dinástica. Distintos personajes, distintos rangos}

A diferencia de otras ciudades mayas (como Piedras Negras o Palenque), los textos de Yaxchilán que registran nombres y acciones de personajes subordinados al k'uhul ajaw comenzaron a utilizarse con más frecuencia a partir del reinado de Yaxuun, a mediados del siglo vil. Diversos investigadores han sugerido que las alusiones y reconocimientos a sajales y demás miembros de la dinastía son parte del "pago" de Yaxuun a estos personajes, que posiblemente lo apoyaron en su lucha para acceder al trono de Yaxchilán. Este argumento no puede ser descartado, pero no debemos olvidar que el registro de personajes con un rango menor en las inscripciones del Clásico maya se generaliza en distintos sitios.

Entre los personajes que mencionan los textos encomendados por Yaxuun encontramos varios sajales, esposas con un rango distinto entre sí, individuos con parentesco político, ajk'uhu'n y ajawtaak menores.

Empecemos con la figura del sajal. David Stuart, en los primeros estudios epigráficos, leyó el título como cahal, pero posteriormente lo cambió a sahal y 


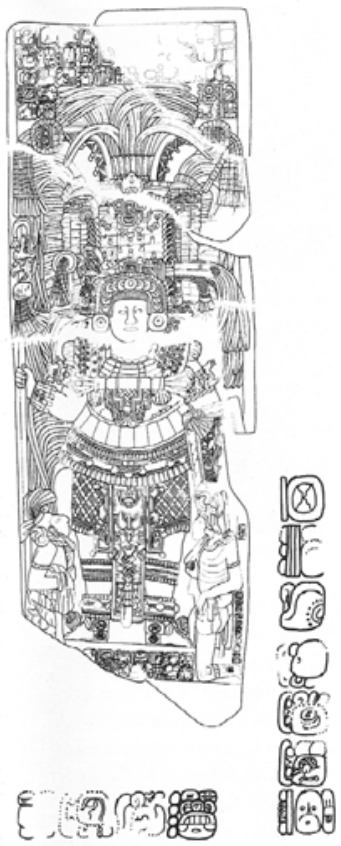

Figura 8. Frente de la Estela 8 de Piedras Negras que muestra el sajal de Itzamnaaj B'ahlam II como prisionero. Tomado de Martin y Grube, 2002 


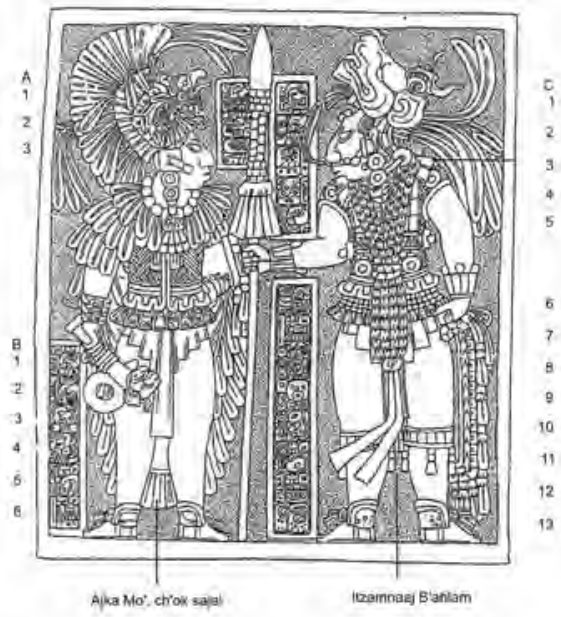

Ficura 9. Dintel del Sitio R. Dibujo de Nikolai Grube

lo tradujo como "el que tiene miedo" (Stuart, 1993) a partir de la raíz yucateca sah. Ahora, con la diferenciación entre la h y la j (Grube, 2004) transcribimos el título como sajal. A partir de estos trabajos, los sajales fueron identificados en eventos militares y como partícipes importantes en ciertos ritos realizados por los gobernantes. Con los avances en el desciframiento de la escritura maya se ha podido detallar la figura del sajal y el rango que tuvo al interior de los señoríos. Péter Bíró (2004) nos dice que la primera mención a un sajal aparece en la región de Piedras Negras a mediados del siglo vII. De manera general, los investigadores opinan que las referencias a sajales inician a partir del crecimiento urbano de las distintas ciudades clásicas. 
Los textos de Yaxuun hablan de varios sajales, pero no todos tenían la misma jerarquía política y social. Un dato interesante lo proporcionan los títulos, los cuales comienzan a ser utilizados por estos personajes, títulos que antes sólo ostentaban los gobernantes de Yaxchilán.

Sin duda, uno de los sajales más importantes durante el reinado de Yaxuun fue K'an Tok Wayib', personaje que aparece en varios monumentos. El Dintel 8 (figura 10), famoso por el análisis de Proskouriakoff (1964), muestra al gobernante Yaxuun B'ahlam y a K'an Tok Wayib' sometiendo a dos prisioneros, "Cráneo Enjoyado" y señor de Kok Te', ${ }^{9}$ respectivamente. En este dintel, K'an Tok Wayib' sólo lleva el título de sajal, pero en el escalón X de la Escalera Jeroglífica 2 (figura 11) es nombrado como b'aah sajal, "primer sajal" o "sajal principal", lo que demuestra que había una clara diferenciación jerárquica entre los sajales. K'an Tok Wayib' aparece en otros dinteles donde también se le identifica como b'aah sajal.

Existen otros tres sajales. Uno de ellos lo encontramos en los monumentos de La Pasadita, un sitio que se ha ubicado sobre el margen derecho del río Usumacinta, en el Petén guatemalteco (Stuart, 1993). En la actualidad se conocen cuatro monumentos de La Pasadita y todos refieren acontecimientos ligados a señores de Yaxchilán. El personaje que ahora nos ocupa (figura 12) también fue representado en algunos textos de Chelte' Chan K'inich, sucesor de Yaxuun. El nombre de este sajal oscila entre Tiloot y Tiloom, porque en algunas ocasiones lleva como complemento fonético un silabograma ma y otras un ta. ${ }^{10}$ En el Dintel 1 de La Pasadita, Tiloot acompaña a Yaxuun en un sacrificio (uchoko'w) de derramamiento realizado en una fecha de final de periodo.

Otro sajal de Yaxuun fue K'in Mo' Ajaw, sajal de tres k'atunes de vida (figura 13). En este monumento, Yaxuun fue representado con un atavío más impresionante que el de su subordinado, pero ambos sostienen un cetro del dios K'awiil, algo que podría sugerir que este sajal en particular fue gobernante o dirigente máximo de alguna ciudad satélite de Yaxchilán. Por lo que hemos visto en los ejemplos anteriores, me parece, de manera tentativa, que sólo K'in Mo' Ajaw fue dirigente de un sitio controlado directamente por Yaxchilán, a diferencia de K'an Tok Wayib' y Tiloot o Tiloom.

El otro personaje asociado con el título de sajal es una de las cuatro esposas de Yaxuun: la señora Chak Joloom (figura 14), madre del siguiente gobernante de Yaxchilán: Chelte' Chan K'inich. Parece que la designación como sajal de la señora Chak Joloom no es casual. Si consideramos que las otras tres esposas de Yaxuun sólo fueron distinguidas con el título de b'aahkab', podemos concluir que quizá la señora Joloom recibió el título de sajal por ser la madre del heredero de Yaxuun.

\footnotetext{
${ }^{9}$ Guillermo Bernal (comunicación personal) propone que el signo silábico ko en algunas ocasiones funciona como el logograma KOK, kok, "tortuga".

${ }^{10}$ Guillermo Bernal (comunicación personal) opina que este cambio en los complementos fonéticos quizá indica la existencia de dos sajales distintos.
} 


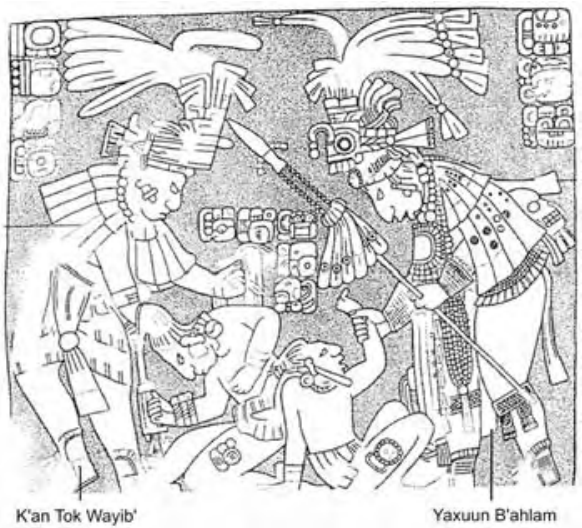

Figura 10. Dintel 8 de Yaxchilán. Dibujo de Peter Mathews, 1997

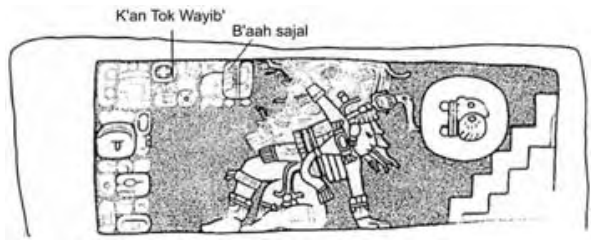

Figura 11. Escalera Jeroglífica II, escalón 10. Dibujo de Peter Mathews, 1997 


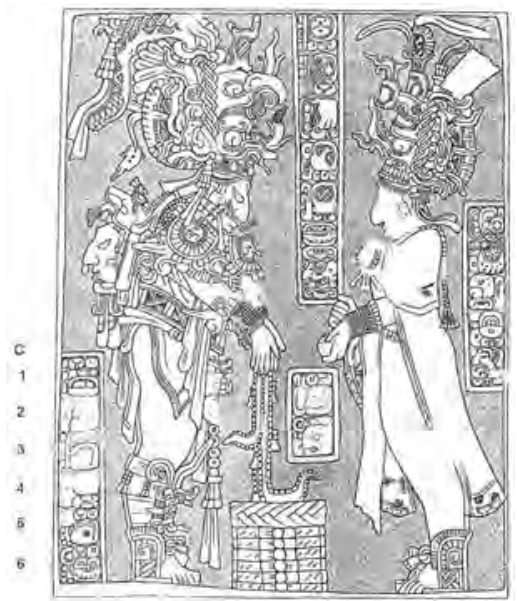

Figura 12. Dintel 1 de La Pasadita. El gobernante Yaxuun B'ahlam IV realiza un sacrificio en presencia del sajal Tuloot. Dibujo de Peter Mathews, 1997

Existe otro personaje en las inscripciones comisionadas por Yaxuun que también se identifica como Chak Joloom. Éste aparece en el último monumento realizado por Yaxuun (figura 15) y se le describe como yichaan ajaw ajwuk b'aak, "tío materno del señor, el de los siete prisioneros". Es decir, el señor Chak Joloom fue tío materno de Chelte' y cuñado de Yaxuun. Aunque Chak Joloom no lleva el título de sajal, sin duda alguna jugó un papel importante al interior del señorío. En el Dintel 58 (figura 16) comparte escena con su sobrino Chelte'. Los primeros glifos del monumento lo identifican como ajwuk joloom?, ${ }^{11}$ quizá el mismo título

"El logograma colocado en A1 del Dintel 58 no ha sido descifrado. Sin embargo, por la similitud de la información del Dintel 9 y del Dintel 58 como un título de Chak Joloom, sugiero la lectura Joloom, aunque es tentativa. 


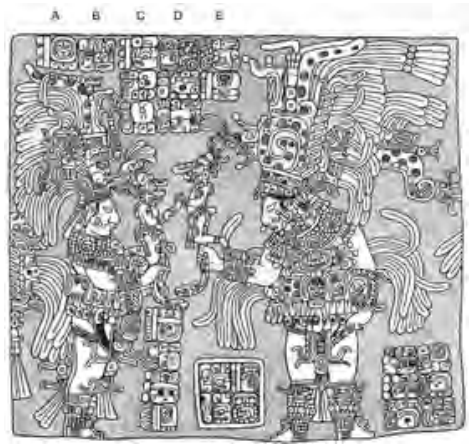

FicurA 13. Yaxuun B'ahlam (a la derecha) sostiene un cetro del dios K'awiil al igual que el sajal K'in Mo' ajaw. Dintel 3 de Yaxchilán. Dibujo de Peter Mathews, 1997

que recibe en el Dintel 9 de "señor de los siete prisioneros" o, como parece ser el caso del Dintel 58, "el de los siete cráneos".

Por otro lado, el glifo leído como ajk'uhu'n, que identifica a ciertos personajes en las inscripciones mayas, aún no se ha traducido del todo. Linda Schele y Mary Miller fueron las primeras en proponer una lectura y una traducción sugiriendo que el glifo se leía "él, de la sangre" (1986). Después hubo varios estudiosos que se internaron en su significado sin que hubiera un consenso. Sin embargo, la traducción de "el que guarda las cosas sagradas" se ha utilizado en los últimos años (Jackson y Stuart, 2001). No hay duda de que los personajes asociados con este título desempeñaron funciones diferentes a los sajales, quienes se han identificado como dirigentes políticos y militares de los señoríos. Además, los epigrafistas han señalado que los ajk'uhu'n debieron tener un lugar privilegiado en la corte y conocimientos de escritura (Stuart, 1993; Jackson y Stuart, 2001; Bíró, 2004; Bernal Romero y Venegas Durán, 2005).

A partir de la identificación del título de ajk'uhu'n se ha establecido que estuvo asociado con individuos que debieron tener una función esencialmente religiosa (Maricela Ayala, comunicación personal), por lo que Bernal Romero y Venegas Durán (2005: 11) proponen que “|...| los dignatarios k'uhu'n pueden ser 


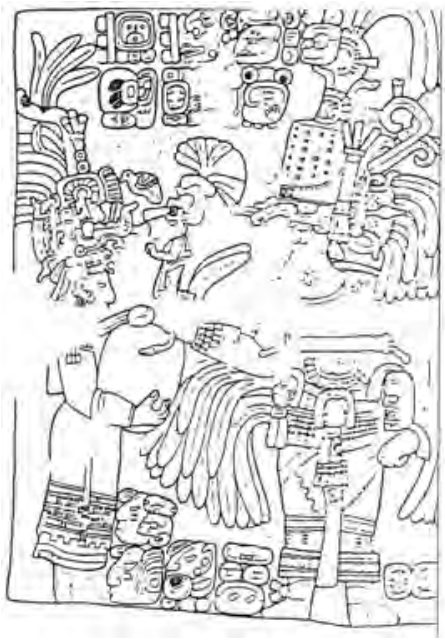

Figura 14. La señora Chak Joloom sostiene entre sus manos un bulto ceremonial. Los glifos inferiores la identifican como sajal. Dintel 54 de Yaxchilán. Dibujo de Peter Mathews, 1997

comprendidos como consejeros muy cercanos del k'uhul ajaw y ministros del culto específicamente funerario. Bajo esta percepción, la acepción del título como El que adora o venera quizá resulte más viable". Su propuesta se argumenta en la lápida del Templo de las Inscripciones, donde a dos personajes se les identifica con este título.

En los textos de Yaxchilán tenemos el glifo ajk'uhu'n en el Dintel 19 pero, debido a su terrible erosión, no se puede especificar el contexto en el cual aparece. Otra mención de este título la tenemos en uno de los dinteles del Sitio R (figura 17) asociado con un personaje que comparte escena con Yaxuun, quien baila con 


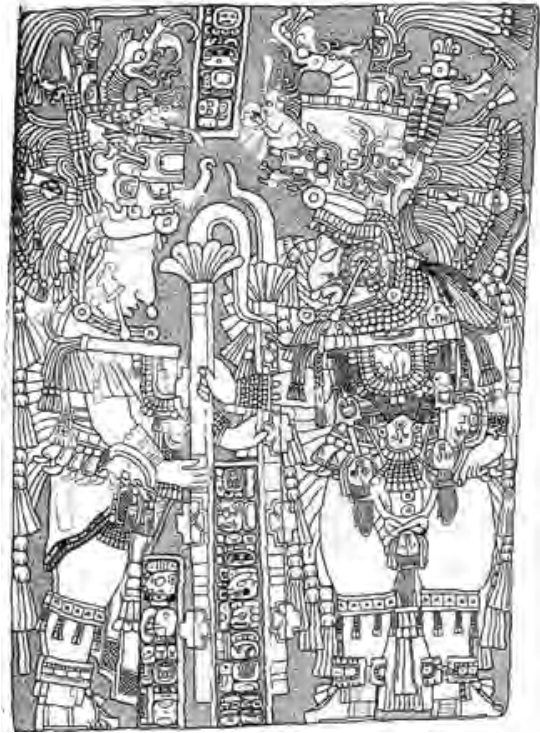

Ficura 15. El señor Chak Joloom (a la izquierda) intercambia "bastones alados" con Yaxuun B'ahlam. Dintel 9 de Yaxchilán. Dibujo de Peter Mathews, 1997

"la serpiente de las visiones" (Grube, 2006). Este monumento presenta algunas características iconográficas y epigráficas extrañas o, por lo menos, diferentes a lo que encontramos en otras esculturas. En primer lugar, resalta la postura de baile de Yaxuun. Tenemos varios dinteles y estelas de Yaxchilán que nos narran distintas danzas realizadas por gobernantes y personajes principales, pero sólo 


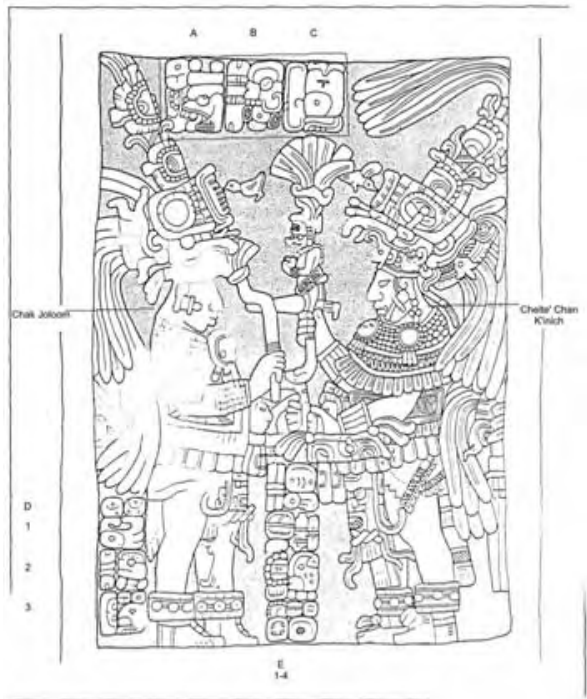

Figura 16. El texto del lado izquierdo identifica a Chak Joloom como "tío materno" de Chelte' Chan K'inich (a la derecha). Dibujo de Peter Mathews, 1997

se registra en el texto a través del verbo ahk'taj, "bailó", y la iconografía no hace gráfica la danza. Aunque en monumentos de otros sitios (como Quiriguá y Palenque) los personajes son representados bailando, en Yaxchilán, y sobre todo en las esculturas de Yaxuun, esto nunca ocurre. En segundo lugar, el texto asociado con el personaje que acompaña a Yaxuun, identificado como ajk'uhu'n, dice uchaan ukan, "el guardián de la serpiente de". Guillermo Bernal (comunicación 


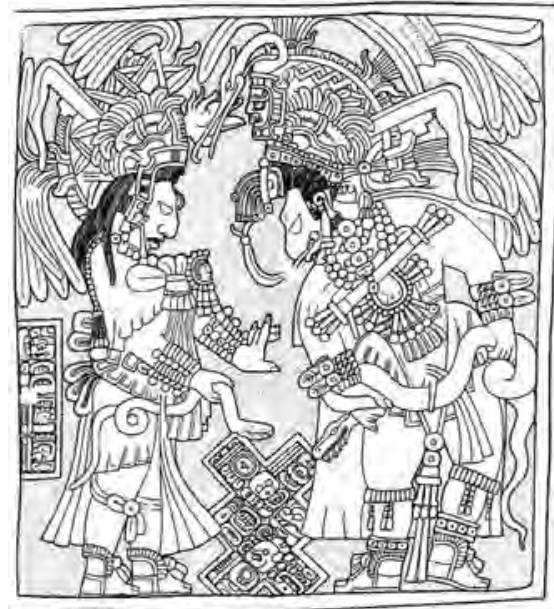

Figura 17. Dintel del Sitio R donde Yaxuun B'ahlam baila con "la serpiente de las visiones". Dibujo de Nikolai Grube

personal) me señaló que la forma de escribir serpiente con los silabogramas ka y na es extraña y no aparece en textos que refieran dicha palabra serpiente, la cual es escrita con el logograma KAN. De todas formas, este monumento ha sido considerado por los epigrafistas en sus reconstrucciones; por ello se analizará en este trabajo.

El nombre del ajk'uhu'n es Ajkamo', y es "el supervisor de la serpiente de". El título de supervisor, observador (uchaan) de la serpiente que se relaciona con el ajk'uhu'n quizá hace alusión a la serpiente con la que Yaxuun realizó el rito de danza y permite confirmar el desempeño en el ámbito religioso de los ajk'uhu'n. 
En cuanto al registro en los textos de ajawtaak menores, éstos fueron escasamente representados. En los monumentos de Yaxchilán conocidos no se menciona a ningún ajaw simple, a diferencia del Sitio R (figura 18), donde Yaxuun se impone a un personaje que, de rodillas frente a él pero con vestimenta decorosa que evidencia su rango, fue identificado como Ajka' ?, yajaw de Yaxuun B'ahlam de Yaxchilán. Es interesante la forma como fue representado este personaje. En los monumentos analizados, ninguno de los anteriores individuos demuestra su inferioridad ante el k'uhul ajaw. La distinción entre Yaxuun y sus subordinados se refleja, sobre todo, en un atavío más sencillo por parte de estos últimos. El

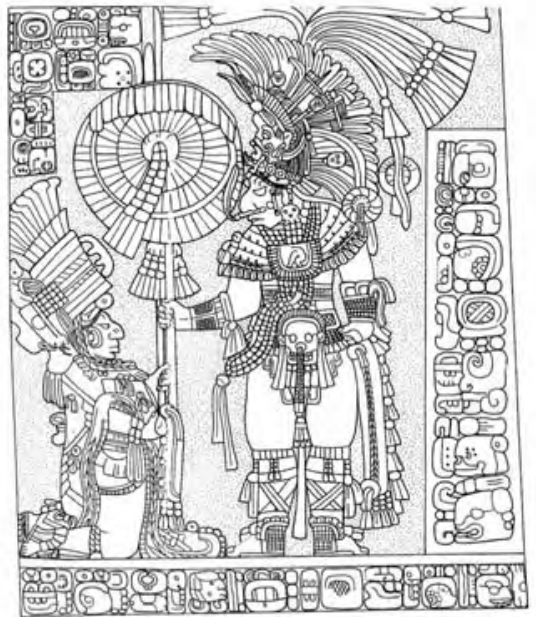

Figura 18. Dintel del Sitio R que muestra la subordinación de un ajaw menor de Yaxuun B'ahlam. Dibujo de Nikolai Grube 
hecho de que Ajka'? aparezca arrodillado ante él (algo muy común en la representación de prisioneros en Yaxchilán) demuestra no sólo un rango menor de los yajawtaak frente a otros personajes, sino también que, probablemente, este individuo fue sometido militarmente.

El último personaje, pero no por ello menos importante, que participó en la alianza renovada por Yaxuun lo encontramos en la cercana ciudad de Bonampak. En los dinteles tempranos de Yaxchilán $(11,35,37,49)$ aparecen mencionados distintos señores de Ak'e (Bonampak), lo que evidencia la presencia de este sitio en la esfera política de Yaxchilán. Sin embargo, después del hiato no vuelve a ser mencionado. Podemos sugerir que Yaxuun se encargó de renovar la alianza con Bonampak a través de una mujer de Pa' Chan, quien aparece en la Estela 2 (figura 19) con el título b'aahkab' y fue esposa del gobernante Yajaw Chan Muwaan. El monumento tiene una fecha (9.17.5.8.9), 6 Muluk 17 Yaxk'in, 11 de junio de 776 d. C. y pudo ser una hija de Yaxuun B'ahlam. Estos lazos entre Yaxchilán y Bonampak se harán más evidentes durante el reinado de Chelte' Chan K'inich.

\section{El escenario político}

Después de diez años de interregno, Yaxchilán vive uno de los periodos más fructíferos de su historia. Yaxuun construyó y amplió varios conjuntos arquitectónicos de la ciudad y erigió más de la mitad de los monumentos que conocemos hasta ahora. En un afán de "legitimación", como han considerado algunos autores, Yaxuun nos permite hoy conocer un poco más la composición dinástica del señorío durante su reinado. Aunque numerosos investigadores han señalado la proliferación de alusiones a personajes menores en los textos jeroglíficos de las ciudades mayas, es importante decir que en Yaxchilán, a diferencia de otros sitios, este fenómeno disminuye con la muerte de Yaxuun y el reinado de Chelte', de tal suerte que el último monumento de Yaxchilán, el Dintel 10, no registra a sajales, ajk'uhu'n ni ajawtaak simples.

Los monumentos erigidos en el reinado de Yaxuun, por lo tanto, son importantes para analizar y comprender la organización sociopolítica de Yaxchilán a mediados del siglo vil. Esta diversidad de personajes que convergieron en un escenario político común muestra la gran complejidad dinástica que debió existir al interior de los señoríos mayas de la época Clásica y evidencia la jerarquización de los distintos espacios políticos (Izquierdo y de la Cueva, 2004). El estudio de los diversos actores no sólo revela esta pluralidad, también permite conocer los cargos, las jerarquías y las funciones que desempeñaron.

Stuart (1993) ha señalado que la mención explícita en los textos a eventos militares y de guerra se generaliza en la zona de las tierras bajas mayas a partir del siglo vil. Como consecuencia, o quizá de forma paralela, los textos jeroglíficos comienzan a abrirse y ya no se ocupan solamente de narrar acontecimientos en la vida del k'uhul ajaw. Hay monumentos que presentan como actores únicos a 


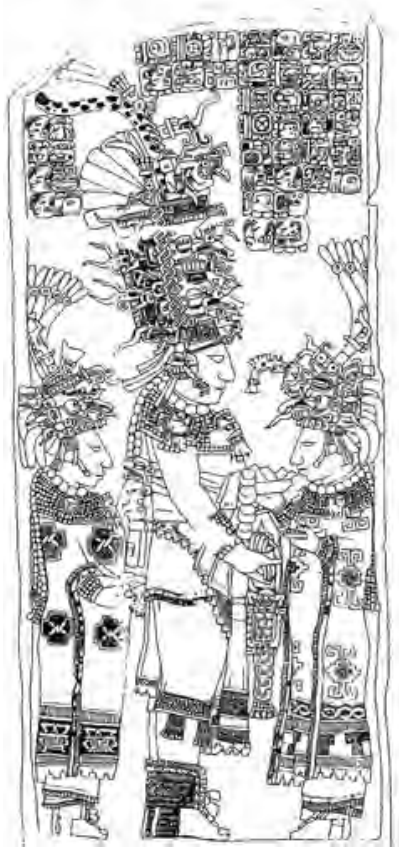

Figura 19. Al centro se encuentra el gobernante Yajaw Chan Muwaan y, detrás de él, una señora de Yaxchilán. Dibujo de Peter Mathews, 1997 
personajes subalternos no sólo en la escritura, sino también en la iconografía. Es indudable que Yaxuun pudo extender sus dominios y afianzar relaciones con otros sitios a través de la nobleza subordinada.

También encontramos cambios en el ámbito militar. La guerra jugó un papel importante en la expansión y consolidación del poder durante el reinado de Yaxuun. El primer cautivo mencionado en sus textos (Dintel 16) es un sajal del señor de Wakab', un sitio que en la actualidad se ha identificado con Santa Elena (ver figura 1) y que no fue mencionado por los gobernantes anteriores de Yaxchilán. Además, una de las esposas de Yaxuun, la señora Mut B'ahlam, pertenecía al señorío de Hix Witz. Recordemos que tanto Yaxuun B'ahlam III como Itzamnaaj B'ahlam II mencionan personajes de este sitio como cautivos en las Escaleras Jeroglíficas. Estos registros antagónicos desaparecen con el reinado de Yaxuun, algo que nos permite apreciar el cambio en las alianzas y disputas.

Volviendo al segmento sajal, éste participó no sólo en ritos y conmemoraciones realizadas por el gobernante de Yaxchilán; también fue una pieza fundamental en las actividades bélicas y de gobierno regional. Lo anterior se ha interpretado como la distribución del poder entre los integrantes de las dinastías mayas y como un cambio en la organización sociopolítica interna de las ciudades. Bernal (2003) ha concluido que uno de los últimos monumentos de Palenque, el Tablero de K'an Tok, revela la forma en que K'inich K'uk' B'ahlam III, a través del reconocimiento a sus sacerdotes, intentó ratificar el apoyo y la lealtad para conseguir un fin político: el fortalecimiento del señorío de Palenque el cual, de la misma forma que las demás ciudades mayas clásicas, comenzaba a desintegrarse.

Aunque los textos de Yaxuun analizados en el presente trabajo no omiten la presencia del k'uhul ajaw frente a eventos de señores subalternos (como ocurre en Piedras Negras, Lacanjá y El Cayo), me parece que la crisis dinástica y política que comenzaba a amenazar a los señoríos a partir del siglo vil comenzó en Yaxchilán desde el momento del interregno, cuando Yaxuun, junto con otros personajes en igualdad política y social, comenzó a luchar hasta conseguir el trono.

La figura y el reinado de Yaxuun B'ahlam IV aún presentan interrogantes que requieren de un trabajo más amplio. En esta ocasión quise mencionar algunos aspectos de sus monumentos, donde encontramos claras diferencias con los textos anteriores y que son muestra no sólo de lo que ocurre en Yaxchilán, sino en toda la zona maya, donde vemos que los textos comienzan a hablarnos de personajes que antes eran mencionados en un nivel secundario.

Debido a que esta pluralidad de jerarquías políticas en los textos de Yaxuun sólo se encuentra en su reinado y no se prolonga a los siguientes gobernantes de Yaxchilán, la idea de los epigrafistas (Stuart, 1993; Mathews, 1997; Grube, 1999; Grube y Martin, 2002; Bíró, 2004) de que Yaxuun agradeció el apoyo de distintos personajes para ascender al trono aún resulta vigente. Los problemas que debió enfrentar al momento del fallecimiento de su padre, Itzamnaaj B'ahlam II, se evidencian con la representación que hace de la nobleza subordinada, la cual pugnó por mayores privilegios. 


\section{BIBLIOGRAFÍA}

Aulie, H. Wilbur y Evelyn W. de Aulie

1978 Diccionario ch'ol-español, español-ch'ol. México: Secretaria de Educación Pública / Instituto Linguiístico de Verano (Serie de Vocabularios y Diccionarios Indígenas Mariano Silva y Aceves, 21).

Ayala Falcón, Maricela

1985 El fonetismo en la escritura maya. México: unam, ufl, Centro de Estudios Mayas (Cuadernos, 17).

2002 El bulto ritual de Mundo Perdido, Tikal. México: unam, IfL, Centro de Estudios Mayas.

2002 "Pero Chubo guerra en Toniná? O de cómo las apariencias engañan", Estudios de Cultura Maya, XXII: 151-166. México: unaM, HFL, Centro de Estudios Mayas.

Barrera Vázquez, Alfredo (coord.)

1990 Diccionario Maya Cordemex. México: Porrúa.

Bernal Romero, Guillermo

2003 "El Tablero de K'an Tok. Reconstrucción, análisis epigráfico e implicaciones historiográficas de una inscripción glífica maya del Grupo XVI, Palenque, Chiapas". Tesis de licenciatura en Historia. México: unam, Facultad de Filosofía y Letras.

Bernal Romero, Guillermo y Benito Jesús Venegas Durán

2005 "Las familias de Palenque. Poder dinástico y tejido social del señorío de B'aakal durante el periodo Clásico Tardío", Lakamha'. Boletín informativo del museo de sitio y zona arqueológica de Palenque, 4 (16, julio-septiembre): 913.

Bíró, Péter

2004 "La organización política del Clásico maya (200-900 d.C.): la ciudad de Piedras Negras". Tesis de maestría en Estudios Mesoamericanos. México: unam, Facultad de Filosofía y Letras.

Brokmann, Carlos

2000 Tipología y análisis de la obsidiana de Yaxchilán, Chiapas, México: INAH (Colección Científica).

Cuevas García, Martha y Guillermo Bernal Romero

2002 "La función ritual de los incensarios compuestos del Grupo de las Cruces de Palenque", Estudios de Cultura Maya, XXII: 13-32. México: unam, Iㅏ, Centro de Estudios Mayas.

Fitzsimmons, James L.

2005 "Kings of Jaguar Hill: Monuments and Caches at Zapote Bobal". Informe de investigación entregado a FaMsı (www.famsi.org/reports/05047/index.html). 
García Moll, Roberto

2003 La arquitectura de Yaxchilán. México: Plaza y Valdés.

2004 "Yaxchilán: evolución interna", Homenaje a Jaime Litvak, Antonio Benavides, Linda Manzanilla y Lorena Marambell (coords.). México: INAH/Conaculta/UnAM, Instituto de Investigaciones Antropológicas.

Garza Camino, Mercedes de la

2002 "El puesto del gobernante en el cosmos y sus ritos de poder", Estudios de Cultura Maya, XXII: 247-259. México: UnAM, IIFL, Centro de Estudios Mayas.

Graham, lan

1982 Corpus of Maya Hieroglyphic Inscriptions, volumen 3, parte 3, Yaxchilán. Cambridge [Massachusetts]: Peabody Museum of Archaeology and Ethnology, Harvard University Press.

Grube, Nikolai

1999 "Observations on the Late Classic Interregnum at Yaxchilan", The Archaeology of Mesoamerica, pp. 116-127, Linda Manzanilla (ed.). Londres: Cambridge Univesity Press.

2004 "The Orthographic Distinction between Velar and Glottal Spirants in Maya Hieroglyphic Writing", The Linguistics of Maya Writing, pp. 61-81. S. Wichmaann (ed.). Salt Lake City: The University of Utah Press.

2006 "Los orígenes del sistema de escritura", Segundo curso-taller de escritura jeroglífica maya, María Eugenia Guitiérrez González (trad.). México: unam, Facultad de Filosofia y Letras, Posgrado en Estudios Mesoamericanos.

Houston, Stephen D., et al.

2006 "Levantamiento preliminar y actividades de registro en El Zotz, Biotopo región San Miguel la Palotada, Petén", informe entregado al Departamento de Monumentos Prehispánicos y Coloniales, Dirección General del Patrimonio Cultural y Natural de Guatemala.

Izquierdo y de la Cueva, Ana Luisa

2004 "Unidad y fragmentación del poder entre los mayas", Estudios de Cultura Maya, XXV: 57-76. México: UNAM, IFF., Centro de Estudios Mayas.

Jackson, Sarah y David Stuart

2001 "The Aj K'uhun Title: Deciphering a Classic Maya Term of Rank", Ancient Mesoamerica, 1(2): 217-228. Cambridge [Reino Unido]: Cambridge University.

Martin, Simon y Nikolai Grube

2002 Crónica de los reyes y reinas mayas. La primera historia de las dinastías mayas, traducción de Lorenzo Ochoa. México: Planeta.

Martin, Simon

2004 "A Broken Sky: The Ancient Name of Yaxchilan as Pa' Chan", PARI Journal, 5 (1):1-7. California: Precolumbian Art Research Institute. 
Mathews, Peter

1997 La escultura de Yaxchilán, traducción de Antonio Saborit. México: INAH (Serie Arqueología).

Proskouriakoff, Tatiana

1950 A Study of Classic Maya Sculpture. Washington D.C.: Carnegie Institution of Washington (Publication 593).

1963 "Historical Data in the Inscriptions of Yaxchilan (I)", Estudios de Cultura Maya, III: 149-168. México: UNaM, IIFL, Centro de Estudios Mayas.

1964 "Historical Data in the Inscriptions of Yaxchilan (II)", Estudios de Cultura Maya, IV: 177-202. México: unAm, IIFL, Centro de Estudios Mayas.

Sotelo Santos, Laura Elena

1992 Yaxchilán. México: Gobierno del Estado de Chiapas.

Stuart, David

"Historical inscriptions and the Maya collapse", en Lowland Maya Civilization in the Eighth Century A.D., J. A. Sabloff y J. S. Henderson (eds.), pp. 321-354. Dumbarton Oaks Research Library and Collection, Washington, D.C.

Tate, Carolyn E.

1987 "The Royal Women of Yaxchilan", Memorias del primer Coloquio Internacional de Mayistas, 5-10 de agosto de 1985. México: unam, HFL, Centro de Estudios Mayas.

Thompson, J. Eric

1962 A Catalog of Maya Hieroglyphs. Norman: University of Oklahoma Press. 